\title{
The atherogenic effects of chlamydia are dependent on serum cholesterol and specific to Chlamydia pneumoniae
}

\author{
$\mathrm{He} \mathrm{Hu},{ }^{1}$ Grant N. Pierce, ${ }^{2}$ and Guangming Zhong ${ }^{1,3}$ \\ ${ }^{1}$ Department of Medical Microbiology, \\ ${ }^{2}$ Department of Physiology, and \\ ${ }^{3}$ Division of Stroke and Vascular Disease, St. Boniface General Hospital Research Centre, University of Manitoba, \\ Winnipeg, Manitoba, Canada R3E 0W3 \\ Address correspondence to: Guangming Zhong, Department of Medical Microbiology, University of Manitoba, \\ 508-730 William Avenue, Winnipeg, Manitoba, Canada R3E 0W3. Phone: (204) 789-3835; Fax: (204) 789-3926; \\ E-mail: gmzhong@cc.umanitoba.ca \\ Received for publication July 14, 1998, and accepted in revised form January 20, 1999.
}

Epidemiological investigations have linked Chlamydia pneumoniae infection to atherosclerosis. It is not clear, however, whether C. pneumoniae infection plays a causal role in the development of atherosclerosis. Mice with low-density lipoprotein receptor deficiency were induced to develop atherosclerotic lesions in aorta with a cholesterol-enriched diet that increased serum cholesterol by two- to threefold. Using this mouse model, we found that the chlamydial infection alone with either the C. pneumoniae AR39 or the C. trachomatis MoPn strain failed to induce any significant atherosclerotic lesions in aorta over a period of nine months. However, in the presence of a high-cholesterol diet, infection with the $C$. pneumoniae AR39 strain significantly exacerbated the hypercholesterolemia-induced atherosclerosis, demonstrating that a hypercholesterolemic condition is required for the $C$. pneumoniae to aggravate the development of atherosclerosis. Although both AR39 and MoPn antigens were detected in aorta of mice infected with the corresponding strains, only mice infected with the C. pneumoniae strain AR39 displayed enhanced atherosclerotic lesions, suggesting that the C. pneumoniae species may possess a unique atherogenic property. This study may provide a model for further understanding the mechanisms of C. pneumoniae atherogenesis and evaluating chlamydial intervention strategies for preventing the advancement of atherosclerotic lesions enhanced by C. pneumoniae infection.

J. Clin. Invest. 103:747-753 (1999).

\section{Introduction}

Atherosclerosis is the primary cause of myocardial and cerebral infarctions. Although many risk factors for atherosclerosis have been identified, the etiology of atherosclerosis is still not clear. Among the many identified risk factors, hyperlipidemia is the most common and important factor (1). In addition, recent studies (2-4) have presented compelling evidence suggesting a role for Chlamydia pneumoniae infection in atherosclerosis. First, the prevalence of antibodies to C. pneumoniae in the blood of patients with atherosclerosis is higher than that in control subjects $(5,6)$. Second, studies from various laboratories have reported direct detection of C. pneumoniae in the arteries of patients with atherosclerosis but not in the control arteries, including those damaged after heart transplantation (7-9). Third, in vitro infection of macrophages with $C$. pneumoniae can induce foam-cell formation (10). However, it is still not known whether $C$. pneumoniae infection plays a causal role in atherosclerosis and whether serum cholesterol contributes to the atherogenic effects of C. pneumoniae.

Human infections with chlamydiae are often caused by strains of two chlamydial species, C. trachomatis and C. pneumoniae. The human strains of C. trachomatis cause various ocular and urogenital infections, whereas a mouse strain of C. trachomatis causes mouse pneumonia, which is therefore designated as mouse pneumonitis agent (MoPn). C. pneumoniae is a newly isolated chlamydial species from human respiratory tracts (11). Infection with C. pneumoniae is common, and approximately $50 \%$ of adults worldwide have antibodies to C. pneumoniae (12). Although human respiratory infections with C. pneumoniae have recently been associated with atherosclerosis, ocular and urogenital infections with C. trachomatis have not been indicated in any cardiovascular pathogenesis. It will be interesting to compare the effects of these two chlamydial infections on the development of atherosclerosis, as such a comparison will facilitate the understanding of the precise roles of C. pneumoniae infection in atherosclerosis.

Animal models are often useful tools for evaluating the role of infectious agents in diseases. Although previous studies $(13,14)$ based on rabbit models have provided some information on the involvement of C. pneumoniae infection in atherosclerosis, these studies failed to evaluate the role that serum cholesterol may play in the atherogenesis of C. pneumoniae and failed to address whether the effect on atherosclerosis is specific to the C. pneumoniae species. Mice with low-density lipoprotein receptor knockout (LDLR KO) display increased susceptibility to atherosclerosis $(15,16)$, and this mouse model has been used for studying the pathogenesis of atherosclerosis (17-19). The LDLR KO mice do not develop lesions on a low-cholesterol and low-fat diet. However, a high-cholesterol diet can induce lesions of atherosclerosis in these mice at vascular sites typically affected in human atherosclerosis (16). The LDLR KO mice may, therefore, be suitable for studying 


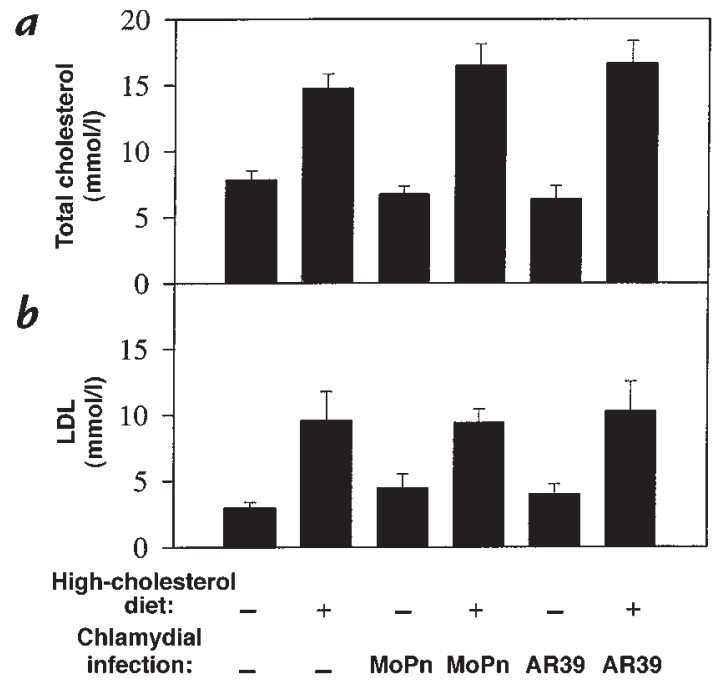

Figure 1

Total cholesterol (a) and low-density lipoprotein ( $\boldsymbol{b}$ ) levels (shown in mean \pm SE along the $y$ axis) in sera of LDLR KO mice as a function of dietary cholesterol supplementation and infection with the two different strains of chlamydia (along the $x$ axis). Regardless of chlamydial infection, mice fed a high-cholesterol diet (high-cholesterol diet alone [ $n=7]$, or plus MoPn [ $n=4]$ or AR39 [ $n=7]$ infection) displayed two- to threefold higher serum total cholesterol $(a)$ or low-density lipoprotein $(b)$ levels than the mice fed a regular mouse chow (normal food without $[n=7]$ or with MoPn $[n=4]$ or AR39 [ $n=7]$ infection). LDLR-KO, low-density lipoprotein receptor knockout.

the role of C. pneumoniae infection in atherosclerosis, as this mouse model can allow both individual and combined assessment of the atherogenic effect of a chlamydial infection and a high-cholesterol diet. In addition, mice are known to be susceptible to a respiratory infection caused by C. pneumoniae intranasal inoculation (20), and C. pneumoniae-induced murine respiratory infection can lead to systemic dissemination (20-22). Finally, the C. trachomatis strain $\mathrm{MoPn}$ is a natural murine respiratory infection agent (23) and can be conveniently used for comparison in the mouse model. Using the LDLR KO mouse atherosclerosis model, we have found that a combination of a high-cholesterol diet with an infection with the AR39 C. pneumoniae strain significantly increased the lesion areas and the lesion severity. Although both AR39 and MoPn antigens were detected in aorta samples from mice infected with the corresponding strains, the C. trachomatis strain MoPn had no atherogenic effect.

\section{Methods}

Organisms. The C. pneumoniae AR39 strain organisms (Washington Research Foundation, Seattle, Washington, USA) were grown in Hep-2 cells (24), and the murine C. trachomatis MoPn strain were grown in HeLa cells as described previously $(25,26)$. The live organisms were purified, aliquoted in a sucrose-phosphate-glutamic acid buffer ( $\mathrm{pH} 7.4)$, and stored at $-80^{\circ} \mathrm{C}$ until used for mouse inoculation.

Experimental design. Forty female B6,129 mice (4-5 weeks old) with LDLR gene deficiency (The Jackson Laboratory, Bar Harbor, Maine, USA) were randomly divided into six groups with five to eight mice in each group. Mice in groups I (seven mice), III (five mice; one died during the experiment), and V (eight mice; one died) were fed with regular mouse chow, whereas mice in groups II (seven mice), IV (five mice; one died), and VI (eight mice; one died) were fed a $2 \%$ cholesterol-supplemented diet (ICN Radiochemicals Inc., Costa Mesa, California, USA). Groups I and II were inoculated intranasally with buffer only. Groups III and IV were inoculated with MoPn organisms at $0.5-1 \times 10^{4}$ inclusion forming units (IFU) per inoculation. Groups V and VI were inoculated with AR39 organisms at $0.5-1 \times 10^{7}$ IFU per inoculation. The inoculation was carried out by dropping a total volume of $15-20 \mu \mathrm{l}$ inocula into one side of the mouse nose under ether. Each mouse was inoculated once monthly for 9 consecutive months. The cholesterol-supplemented diet was initiated on the same day as the first intranasal inoculation. Twenty to 25 days after the final inoculation, all mice were sacrificed for aorta isolation and serum collection. The 9-month observation time point was chosen based on the following pilot experiment, which was conducted before the experiment just described. In the pilot experiment, a total of 20 LDLR KO mice were divided into four groups as just described, except without the two MoPn infection groups, including: the normal mouse chow plus buffer inoculation (four mice) or plus AR39 infection (five mice), and the high-cholesterol diet plus buffer inoculation (five mice) or plus AR39 infection (six mice) groups. Each mouse was inoculated once monthly, and mice from various groups were sacrificed for aortic lesion evaluation at 3, 6, and 9 months after the initial infection.

Morphometric determination of atherosclerosis. Isolation of mouse aorta was carried out based on the method described by Palinski et al. (18). Briefly, mice were anesthetized by intraperitoneal injection with $35 \mathrm{mg} / \mathrm{kg}$ ketamine hydrochloride. After opening the chest cavity, blood was collected by

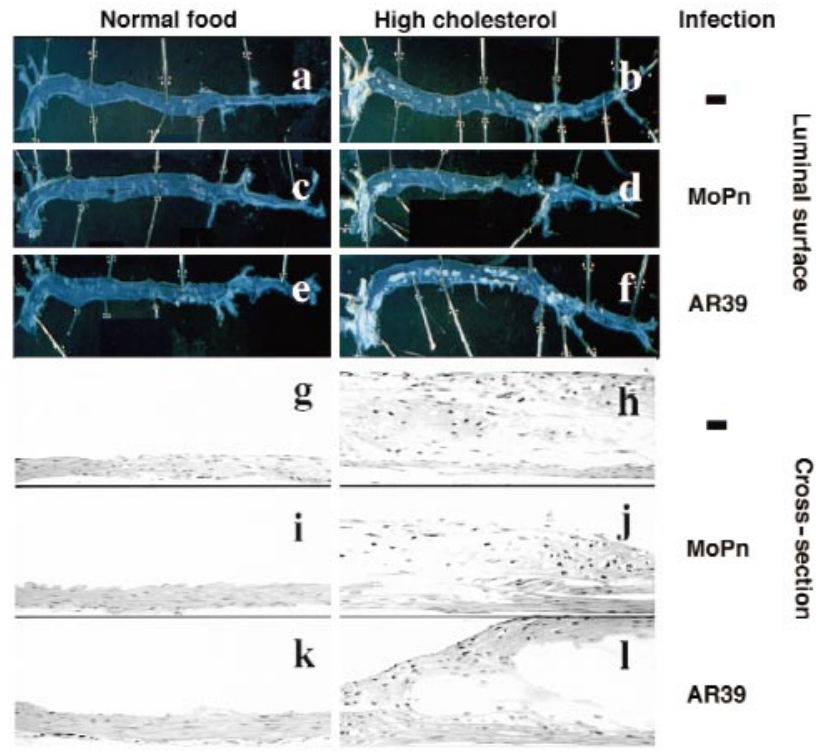

\section{Figure 2}

Representative images of mouse aortic luminal surface (top) and aorta arch cross-sections (bottom) after hematoxylin and eosin staining. Aorta from six groups of mice were isolated for luminal surface lesion observation without any prior staining (panels $\boldsymbol{a}-\boldsymbol{f}$ ). The luminal surface of each entire aorta from aorta arch (left) to the bifurcation (right) was shown. The light-colored atherosclerotic lesions were readily noticeable in aorta from mice fed with a high-cholesterol diet (panels $b, d$, and $f$ ) but not in those of mice fed with a normal food diet (panels $a, c$, and $e$ ). Some of the lesions were further stained with Sudan red for verifying the lipid content (data not shown) after acquiring the images. The aortic arch portions of the samples just described were used to make sections for hematoxylin and eosin staining (panels $g-l$ ) for evaluating lesion severity. The pictures were taken with a Zeiss microscope using a $20 \times$ objective lens. 


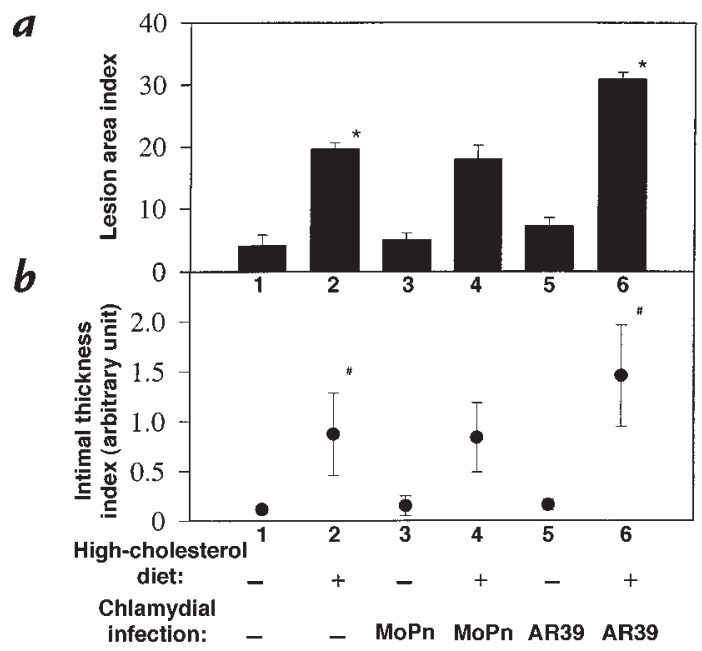

c

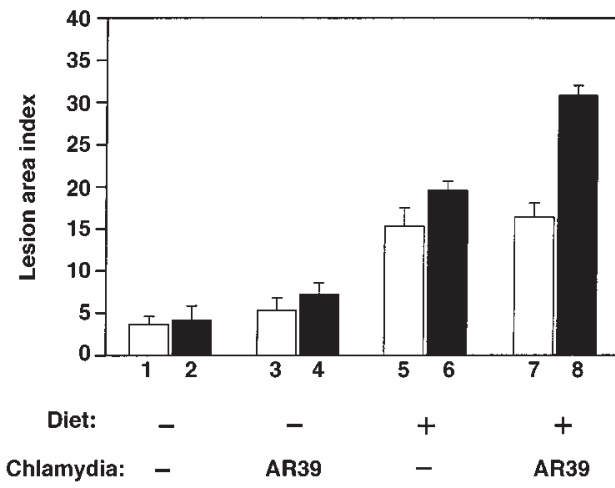

Figure 3

Quantitation of mouse aorta atherosclerosis lesions as lesion area ( $\boldsymbol{a}$ and $\boldsymbol{c}$ ) and intimal thickness index (b). (a) Aorta luminal surface images (as shown in Fig. 2, $a-f$ ) from the six groups of mice, as indicated at the bottom of the present figure and described in Fig. 1 legend, were analyzed using an image software for total aorta luminal surface area and areas covered with lesions. The lesion area index was calculated as percentage of aortic area covered by the atherosclerotic lesions (means $\pm \mathrm{SE}$ ) as shown along the $y$ axis. (b) The cross-sections stained with hematoxylin and eosin as described in Fig. 2, g-l, were used for measuring the lesion intimal thickness. An average of three measurements from three independent sections of the same aorta sample was used as the intimal thickness index (means \pm SE) as shown along the $y$ axis. The AR39 infection plus high-cholesterol group $(n=7)$ is significantly different from the high-cholesterol alone group $(n=7)$ in both lesion area $\left(a ;{ }^{*} P<0.001\right)$ and lesion thickness $\left(b ;{ }^{\#} P<0.05\right)$. (c) Comparison of aortic lesion area index from mice sacrificed at 6 months (open columns; $n=3$ for each group) vs. 9 months (hatched columns; the same data as displayed in $a$ ). For columns 1 vs. 2,1 vs. 3,2 vs. 4,3 vs. 4,5 vs. 6 , and 5 vs. $7, P>0.05$; for column 3 vs. $5, P<0.05$; for columns 4 vs. 6,6 vs. 8 , and 7 vs. $8, P<0.001$.

acupuncture through the left ventricle with a 27-gauge needle. The aorta was then perfused with 3-5 $\mathrm{ml}$ of PBS-EDTA solution (2 mM EDTA in PBS; pH 7.4). After the blood was gently flushed out, the aorta was fixed with $5 \mathrm{ml}$ of formal-sucrose solution (PBS containing $4 \%$ paraformaldehyde, $5 \%$ sucrose, and $2 \mathrm{mM}$ EDTA). After the initial fixation, the aorta and its main branches were dissected from the aortic valve to the iliac bifurcation. The adventitia was removed as much as possible. The isolated aortic trees were opened longitudinally with extremely fine Micropoint Surgical Scissors (Laschal Surgical Inc., Purchase, New York, USA) and pinned flat on a black wax surface. After a further overnight fixation with formal-sucrose and a 12-h rinse in PBS, photographs of the aorta were taken with a Leica MD-2 camera (Heidelberg, Germany) under the exact same conditions of illumination, focal distance, and amplification. The pictures on films were scanned with a Polaroid Sprint Scan film scanner (Polaroid Canada, Rexdale, Canada) to render digitized images. The photographing and digitizing were carried out by Wayne Foster at the University of Manitoba Imaging Services (Winnipeg, Manitoba, Canada). The digitized images displayed a clear difference between the lesion regions and the normal aortic luminal surface. The lesion regions can be clearly traced without difficulty. The size of the lesion areas and total aorta luminal surface areas were then determined in square pixels for each aorta using NIH Image 1.61 software (National Institutes of Health, Bethesda, Maryland, USA). The lesion area index was calculated for each aorta as the ratio of the lesion areas vs. the aorta luminal surface areas multiplied by 100 .

Cross-sections and histology. After photographing, the aorta arch portions were embedded in a Cryoform embedding medium (catalog no. 3383; International Equipment Company, Needham, Massachusetts, USA), and $6-\mu \mathrm{m}$-thick serial sections were prepared using a cryotome (Reichert Scientific Instruments, Buffalo, New York, USA). Three sections, each separated by 10 sections, were stained with hematoxylin and eosin for each aorta arch sample. The first section was always started at the aortic valve. The histological sections were viewed under a microscope, and the images were acquired using an attached video camera. For the purpose of comparison, the exact same conditions were used to acquire each image. The Adobe Photoshop program (Adobe Systems Inc., San Jose, California, USA) was used to measure the maximal intimal thickness, which is defined as the maximal measurement from the lesion luminal surface to the outer surface of the vessel wall. The measurements were expressed as arbitrary units (pixels). Given that the vessel wall is often indistinguishable from the lesion in some lesion areas, the vessel wall was included in the lesion thickness measurement. The maximal intimal thickness was measured for each of the three sections from the same aortic arch sample, and an average of the three measurements was used to represent the maximal intimal thickness for each mouse aorta. It has been shown that the severity of lesions at the origin of the mouse aorta correlated well with that in the entire aorta (19).

Chlamydial antigen staining. Some aortic arch cross-sections were subjected to chlamydial antigen staining. After acetone permeabilization and treatment with $0.2 \% \mathrm{H}_{2} \mathrm{O}_{2}$ to quench the endogenous peroxidase, the sections were stained with a monoclonal antibody against chlamydial LPS (EV1-H1, mouse IgG2a; kindly provided by H. Caldwell at the Rocky Mountain Laboratories, National Institutes of Health, Hamilton, Montana, USA; ref. 27) and a C. pneumoniae-specific antibody (D1E8, mouse IgG1; our unpublished data). The primary antibody binding was probed with a goat anti-mouse IgG conjugated with horseradish peroxidase (Cedarlane Laboratories Ltd., Hornby, Ontario, Canada). An insoluble 4-chloro-1-naphthol substrate (Sigma Chemical Co., St. Louis, Missouri, USA) was used to visualize the antibody binding. Adjacent serial sections from the samples stained positive for chlamydial antigens with the antibodies just described were used for isotype control staining. All stained samples were photographed under a Zeiss microscope (Thornwood, New York, USA) equipped with a differential interference contrast cooled camera using objective lenses of $20 \times$ or $63 \times$.

Serum lipoprotein analysis. Blood was collected from each mouse before aortic perfusion. The sera were used for both anti-chlamydial antibody titration and lipid analysis. The serum total cholesterol, triacylglycerol, and high-density lipoprotein (HDL) was measured with a Hitachi 917 assay system (Boehringer Mannheim Canada, Laval, Quebec, Canada) through a service 


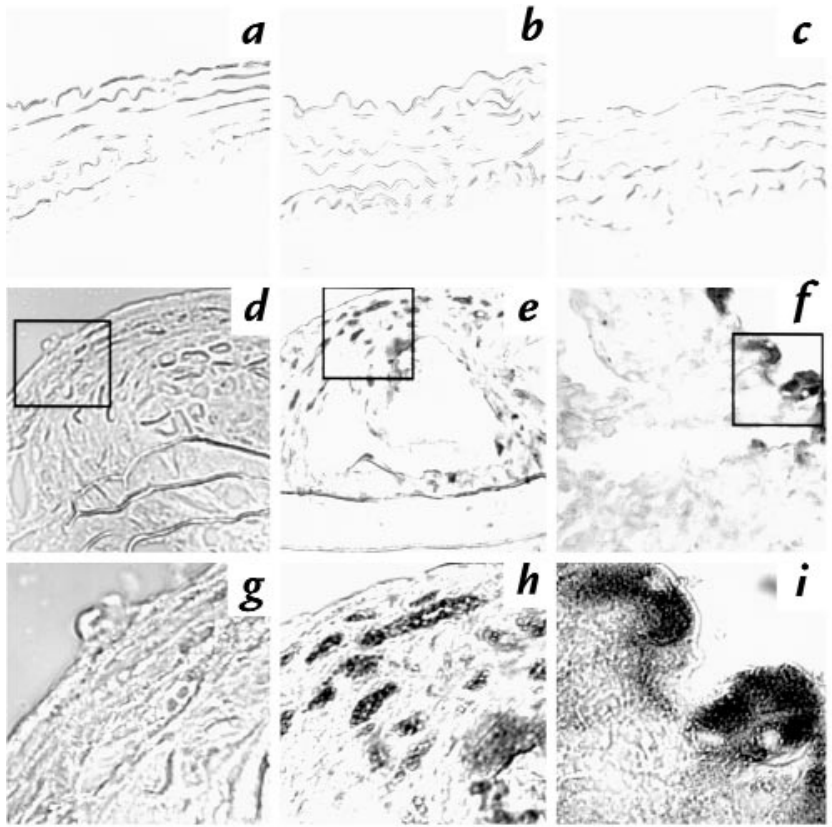

Figure 4

Representative images of chlamydial antigen detected in aorta cross-sections obtained from mice fed a normal food diet $(\boldsymbol{a}-\boldsymbol{c})$ or mice fed a highcholesterol diet $(\boldsymbol{d}-\boldsymbol{f})$. The cross-sections were stained with an anti-chlamydial LPS monoclonal antibody, and the staining was revealed by immunohistochemistry as described in Methods. Images $a$ and $d$ were from mice without chlamydial infection; $b$ and $e$ were from mice infected with MoPn; and $c$ and $f$ were from mice infected with AR39. Images $a-f$ were acquired using a $20 x$ objective lens. Images $g-i$ were acquired under a $63 x$ objective lens from the inset areas in images $d-f$, respectively. Obvious inclusion body-like structures are clearly observed in images $h$ and $i$.

from the Department of Clinical Biochemistry, Health Science Center (Winnipeg, Manitoba, Canada). The low-density lipoprotein (LDL) was calculated as the following: LDL concentration $(\mathrm{mmol} / \mathrm{l})=$ total cholesterol $-(\mathrm{HDL}+$ triacylglycerol $\times 0.46)$.

Titration of chlamydial antigen-specific antibodies in mouse sera. The mouse serum antibodies were measured using the C. pneumoniae AR39 or the C. trachomatis MoPn strain organism-infected HeLa cells as antigens in an immunocytochemistry assay. The chlamydia-infected HeLa cells were allowed to attach to 96-well tissue culture plates, and the attached monolayers were fixed with $4 \%$ paraformaldehyde (Sigma Chemical Co.) followed by a permeabilization with $0.5 \%$ saponin (Sigma Chemical Co.). Antisera serially diluted in PBS were applied to the infected HeLa-cell monolayers. The first antibody binding was detected with a goat anti-mouse IgG conjugated with horseradish peroxidase and an insoluble 4-chloro-1-naphthol substrate. The highest dilution of a given serum that can still reveal obvious chlamydial inclusion bodies was determined as the titer of that serum. The immunocytochemistry assay has been used extensively for detecting chlamydial antigens (4), and the antibody titers obtained using this assay were reproduced using the standard ELISA (25).

Statistical analysis. The Student's $t$ test (unpaired) was used for analyzing the differences between the different animal groups.

\section{Results}

Induction of aortic atherosclerosis in LDLR KO mice with a highcholesterol diet. After treatment for a period of nine months, a total serum cholesterol level of 5-8 $\mathrm{mmol} / \mathrm{l}$ was detect- ed in LDLR KO mice fed a regular mouse chow, whereas mice fed a $2 \%$ cholesterol-supplemented diet exhibited a two- to threefold increase in total serum cholesterol level (Fig. 1a). The total cholesterol was primarily composed of LDL (Fig. $1 b$ and data not shown), which is consistent with previous observations (15). Chlamydial infection with either the C. pneumoniae AR39 or the C. trachomatis MoPn strains did not alter either the total cholesterol or LDL levels in serum. The increase in serum cholesterol level seemed to correlate with aortic atherosclerotic lesion development (Fig. 2). All mice fed a regular mouse chow exhibited a largely normal aortic luminal surface (Fig. 2, panel $a$ ) and normal aortic wall structure on cross-sections (Fig. 2, panel g). However, after being fed a high-cholesterol diet for nine months, all mice developed typical atherosclerosis lesions in the areas of the aortic arch and carotid arteries (Fig. 2, panel $b$ ). Cross-section analysis revealed that these lesions were mainly fatty streaks without an obvious fibrous cap (Fig. 2, panel $h$ ), which is consistent with previous observations (16).

The C. pneumoniae AR39, but not the C. trachomatis MoPn, strain infection significantly enhanced the development of atherosclerotic lesions induced by a high-cholesterol diet. LDLR KO mice fed a regular mouse chow regardless of chlamydial infection displayed a normal aorta luminal surface (Fig. 2, panels $a$, $c$, and $e$ ), and there was no significant difference in the lesion area index between these groups fed normal food (Fig. 3a, columns 1, 3, and 5). Cross-section images of aorta from these groups of mice also revealed normal aortic wall histology (Fig. $2 b$, panels $g, i$, and $k$ ) and minimal intimal thickness index (Fig. 3b, columns 1, 3, and 5). These observations demonstrate that infection alone by either AR39 or MoPn strain is not sufficient to cause any measurable atherosclerotic lesions in aorta of the LDLR KO mice. However, in the presence of a hypercholesterolemia induced by a $2 \%$ cholesterol-supplemented diet, infection with the AR39, but not the MoPn, strain significantly increased the lesion area index from about 20\% (high-cholesterol diet alone group; Fig. $3 a$, column 2) to more than 30\% (AR39 infection plus high-cholesterol treatment group; Fig. $3 a$, column 6) (column 2 vs. $6, P<0.001$ ). The mice treated with a combination of AR39 infection and a high-cholesterol diet developed much more extensive atherosclerotic lesions across the entire aorta luminal surface (Fig. 2, panelf). More importantly, this increase in lesion area was also accompanied by the generation of more severe lesions. Cross-section analysis revealed that AR39 infection in the presence of a high-cholesterol diet induced the development of atherosclerotic lesions with obvious necrotic cores and a fibrous cap (Fig. 2, panel $l$ ). The lesion intima from mice treated with a combination of AR39 infection and a high-cholesterol diet (Fig. 3b, column 6) was also significantly thicker than that in mice fed with a high-cholesterol diet alone (Fig. 3b, column 2) (column 2 vs. 6, $P<0.05$ ). However, mice treated with a combination of MoPn infection and a high-cholesterol diet did not show any significant exacerbation of the atherosclerosis lesions in aorta. There was no significant difference in lesion area (Fig. $3 a$, column 2 vs. 4 ; $P>0.1$ ) and intimal thick- 
ness index (Fig. $3 b$, column 2 vs. $4 ; P>0.1$ ) between the high-cholesterol diet alone group and the group treated with both high-cholesterol diet and MoPn infection.

The nine-month observation time point was chosen based on an earlier pilot experiment in which mice from various treatment groups were sacrificed at three, six, and nine months for aortic lesion evaluation. We found that none of the mice sacrificed at three months displayed any obvious atherosclerosis lesions in their aorta (data not shown). At six months, significant lesions were detected in the aorta of mice fed the high-cholesterol diet. None of the mice fed the normal chow displayed any obvious aortic lesions (Fig. 3c). However, there was no significant difference in lesion area index between the high-cholesterol diet alone and the high-cholesterol diet plus AR39 infection groups $(P=0.852)$. Sera from the AR39-infected mice displayed high titers of anti AR39 organism antibodies (>1:10,000; data not shown), suggesting that these mice were successfully infected with the AR39 organisms. Only by nine months did mice fed the high-cholesterol diet plus AR39 infection display much more severe aortic lesions than the mouse fed the high-cholesterol diet alone (Fig. 3 ). These preliminary results suggest that the high-cholesterol diet initiated the aortic atherosclerotic lesions, whereas the C. pneumoniae infection greatly exacerbated the lesions. However, the relatively small sample size may necessitate further experiments to confirm these results.

Both AR39 and MoPn antigens were detected in aorta of mice infected intranasally with the corresponding chlamydial strains. It is possible that the AR39 or MoPn organisms may have a different capacity to reach the aortic tissue after a respiratory infection. To test the hypothesis, a mouse antichlamydial lipopolysaccharide (LPS) antibody was used to detect the chlamydial antigen in aortic cross-sections. The reasons for choosing this antibody were the following: (a) This antibody can recognize a chlamydial genusspecific epitope on LPS (27). Therefore, both AR39 and MoPn organisms can be detected with the same antibody. (b) LPS epitopes are generally more stable than protein epitopes (28), which provides a more sensitive means to determine the presence of chlamydia in aortic samples. As shown in Fig. 4, chlamydial LPS antigen was detected in aorta from mice treated with a high-cholesterol diet plus infection with either AR39 (Fig. 4, $f$ and $i$ ) or MoPn (Fig. 4, $e$ and $b$ ) strain. The LPS antigen was not detected in mice that did not receive chlamydial infection (Fig. 4, $d$ and $g$ ). The anti-chlamydial LPS antibody staining of the AR39-infected samples was confirmed with C. pneumoniae-specific monoclonal antibody D1E8 (data not shown). The specificity of the staining was further verified by using an isotype-matched control IgG to stain the adjacent serial sections from the same aortic samples that stained positively with the anti-chlamydial LPS antibody. None of the isotype control staining was positive (data not shown). Interestingly, no obvious chlamydial antigen was detected in aortic sections from mice fed a normal mouse chow and infected with either AR39 (Fig. 4c) or MoPn organisms (Fig. 4b). Images taken at a higher power objective lens $(63 \times)$ further revealed chlamydial inclusion body-like structures (Fig. $4 h$ for MoPn; Fig. $4 i$ for AR39 inclusion bodies). These images also showed that multiple distinct inclusion body-like structures were

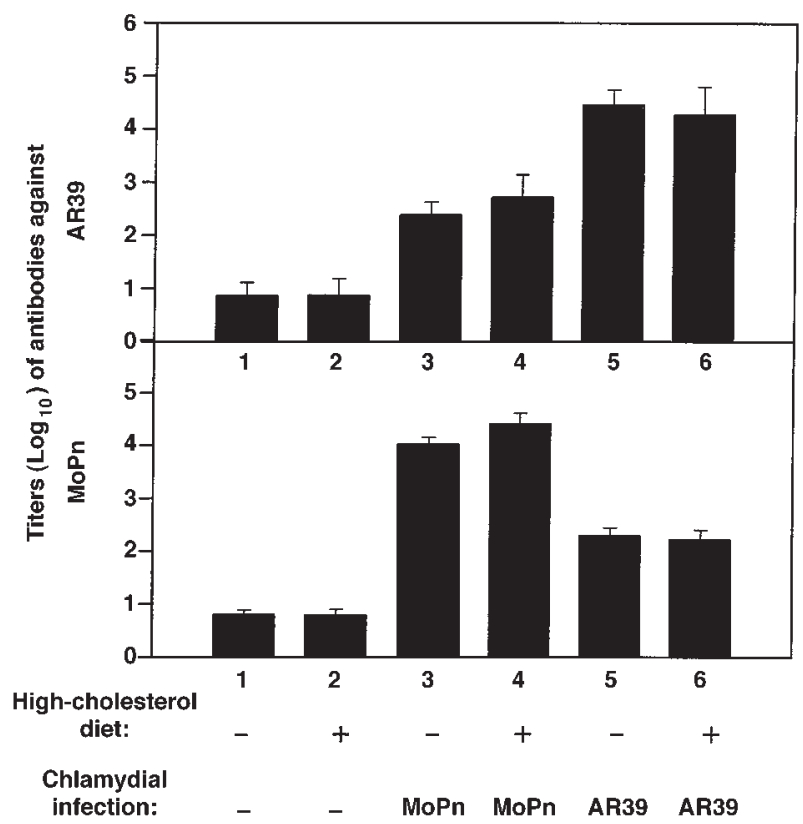

Figure 5

Titration of serum antibodies against chlamydial MoPn (a) and AR39 (b) organisms in mice fed a normal or cholesterol-supplemented diet with or without chlamydial infection as indicated at the bottom of the figure and described in Fig. 1 legend.

found across the lesion tissues from mice infected with MoPn (Fig. 4, $e$ and $b$ ). Intensive smear staining was mainly found beneath the endothelial layer of the lesion tissues from mice infected with AR39 organisms (Fig. 4, $f$ and $i$ ). In all, samples from three of the seven mice treated with both a high-cholesterol diet and AR39 infection and four of the four mice treated with both high-cholesterol diet and MoPn infection were stained positive for chlamydial LPS antigen. None of the rest of the mouse samples stained positive.

We next compared antigen-specific immune responses to MoPn and AR39 strain infection in these mice. The titers of serum antibodies were evaluated against both MoPn and AR39 antigens. As shown in Fig. 5, mice infected with AR39 regardless of the diet used produced antibodies preferentially recognizing AR39 antigens with an average titer of $>1: 10,000$ (Fig. $5 a$, columns 5 and 6). Although the antibodies produced by the AR39infected mice also bound to MoPn antigens, the titer was significantly lower (1:1,000 or less; Fig. 5b, columns 5 and 6). Conversely, mice infected with MoPn displayed MoPn-specific antibodies at about 1:10,000 (Fig. 5b, columns 3 and 4) and AR39-specific antibodies at 1:1,000 or less (Fig. 5a, columns 3 and 4). The background reactivity of the serum from mice without prior exposure to chlamydial organisms was detected at about 1:20 dilution (Fig. 5, $a$ and $b$, columns 1 and 2). These observations suggest that a strain-specific antibody production was predominant in the LDLR KO mice. This is consistent with previous observations (29) that antibodies are produced mainly to strain-specific epitopes when hosts are infected with live chlamydial organisms. Interestingly, the antibody titers in mice fed either a 
normal mouse chow or a high-cholesterol diet are similar (Fig. 5, $a$ and $b$, column 3 vs. 4 and 5 vs. 6 ), suggesting that the hypercholesterolemia did not affect mouse antibody production to chlamydial antigens.

\section{Discussion}

Although C. pneumoniae infection has been associated with atherosclerosis (2), the precise role of C. pneumoniae infection in the development of atherosclerosis is still not clear (30). By using the LDLR KO mouse atherosclerosis model, we have demonstrated that hypercholesterolemia may be a necessary factor for the C. pneumoniae infection to induce an exacerbation of atherosclerotic lesions. Infection with the C. pneumoniae AR39 strain alone did not induce any obvious atherosclerosis lesions. The stimulation of atherosclerosis was not due to the AR39 causing an increase in serum cholesterol levels. It is presently unclear how serum cholesterol interacts with the AR39 infection to induce accelerated atherosclerosis. No obvious chlamydial antigens were detected in aortic sections from mice infected with chlamydia but maintained on a normal diet. This suggests that the initial lesion induced by the high-cholesterol diet may be necessary for the C. pneumoniae to infect and reside in the aortic tissues successfully. Comparison of the aortic lesions between mice sacrificed at different times after the initial treatment suggested that the high-cholesterol diet initiated the early lesion formation and the AR39 infection exacerbated the lesion advancement later. However, these observations were made on a relatively small sample size and will require further study.

Interestingly, the stimulatory effect on vessel lesions was only observed in mice infected with the C. pneumoniae strain AR39 but not with the C. trachomatis strain MoPn organisms. This suggests that the C. pneumoniae AR39 strain may possess a unique property for the atherosclerotic exacerbation. It is unknown what is responsible for this strain-specific difference. However, this study has provided the following evidence to exclude several possibilities regarding the differential effect of AR39 infection: (a) The AR39 infection-induced exacerbation of atherosclerosis was not likely due to a skewed immune response induced by the AR39 organisms, as the infected mice mounted an equivalent antibody response to the infection with either AR39 or MoPn organisms. A dominant strain-specific antibody response was generated to the chlamydial infection by the LDLR KO mice, which is consistent with previous observations made in other hosts (29). (b) Differential migration of the chlamydial species to aortic tissues may not be a factor. Both AR39 and MoPn antigens were detected in aorta tissues, but only AR39 infection exacerbated the atherosclerosis lesions. Because the observations that C. pneumoniae antigens were detected in the aorta from patients with atherosclerosis $(3,31)$, it has been thought that migration to aortic tissue may be a unique property of C. pneumoniae that contributes to its role in atherosclerosis. However, we demonstrated that not all infectious agents found in aortic tissues can induce or exacerbate atherosclerosis lesions. Although, to our knowledge, this is the first report to detect MoPn antigen in aortic tissue, it has been demonstrated previously that respiratory infection of mice with MoPn can lead to the dissemination of the organisms into spleen tis- sues (23). However, other independent detection assays will be required to further confirm the presence of MoPn organisms in these aortic tissues.

In conclusion, the results of this study not only demonstrated that infection with C. pneumoniae can directly stimulate atherogenesis but also, more importantly, provided novel information regarding the mechanisms of chlamydial involvement in vascular diseases. The chlamydial atherogenic effects were dependent on the presence of elevated serum cholesterol. Our results further suggested that not all infectious agents detected in the vessel lesions contributed to the lesion advancement and that C. pneumoniae may possess a unique biologic property for its atherogenesis. One potential weakness of this study that may limit our conclusions is the small sample size used over a relatively long experimental period. Variability in results is possible in such experiments. The findings should be considered as preliminary in nature but of sufficient interest to stimulate further study on this topic using this and other models.

\section{Acknowledgments}

We thank Wayne Foster for assistance in acquiring the digitized images and Dongji Zhang for assistance in mouse intranasal inoculation during the initial stage of the study. This work was supported by grants from the Heart and Stroke Foundation of Manitoba and the Medical Research Council of Canada. G. Zhong is a Scholar of the Medical Research Council of Canada.

1. Dahlen, G.H., and Stenlund, H. 1997. Lp(a) lipoprotein is a major risk factor for cardiovascular disease: pathogenic mechanisms and clinical significance. Clin. Genet. 52:272-280.

2. Mlot, C. 1996. Chlamydia linked to atherosclerosis. Science. 272:1422.

3. Saikku, P. 1997. Chlamydia pneumoniae and atherosclerosis-an update. Scand. J. Infect. Dis. Suppl. 104:53-56.

4. Grayston, J.T., et al. 1995. Chlamydia pneumoniae (TWAR) in atherosclerosis of the carotid artery. Circulation. 92:3397-3400.

5. Saikku, P., et al. 1988. Serological evidence of an association of a novel Chlamydia, TWAR, with chronic coronary heart disease and acute myocardial infarction. Lancet. 2:983-986.

6. Puolakkainen, M., et al. 1993. Serological response to Chlamydia pneumoniae in adults with coronary arterial fatty streaks and fibrolipid plaques. J. Clin. Microbiol. 31:2212-2214.

7. Ong, G., Thomas, B.J., Mansfield, A.O., Davidson, B.R., and TaylorRobinson, D. 1996. Detection and widespread distribution of Chlamydia pneumoniae in the vascular system and its possible implications. J. Clin. Pathol. 49:102-106.

8. Kuo, C.C. 1995. Chlamydia pneumoniae (TWAR) in coronary arteries of young adults (15 to 34 years old). Proc. Natl. Acad. Sci. USA. 92:6911-6914.

9. Ramirez, J.A. 1996. Isolation of Chlamydia pneumoniae from the coronary artery of a patient with coronary atherosclerosis. The Chlamydia pneumoniae/Atherosclerosis Study Group. Ann. Intern. Med. 125:979-982.

10. Kalayoglu, M.V., and Byrne, G.I. 1998. Induction of macrophage foam cell formation by Chlamydia pneumoniae. J. Infect. Dis. 177:725-729.

11. Grayston, J.T., et al. 1990. A new respiratory tract pathogen: Chlamydia pneumoniae strain TWAR. J. Infect. Dis. 161:618-625.

12. Grayston, J.T., Diwan, V.K., Cooney, M., and Wang, S.P. 1989. Community-and hospital-acquired pneumonia associated with Chlamydia TWAR infection demonstrated serologically. Arch. Intern. Med. 149:169-173.

13. Muhlestein, J.B., et al. 1998. Infection with Chlamydia pneumoniae accelerates the development of atherosclerosis and treatment with azithromycin prevents it in a rabbit model. Circulation. 97:633-636.

14. Fong, I.W., et al. 1997. Rabbit model for Chlamydia pneumoniae infection. J. Clin. Microbiol. 35:48-52.

15. Ishibashi, S., et al. 1993. Hypercholesterolemia in low density lipoprotein receptor knockout mice and its reversal by adenovirus-mediated gene delivery. J. Clin. Invest. 92:883-893.

16. Breslow, J.L. 1996. Mouse models of atherosclerosis. Science. 272:685-688.

17. Bird, D.A., et al. 1998. Effect of probucol on LDL oxidation and atherosclerosis in LDL receptor-deficient mice. J. Lipid Res. 39:1079-1090.

18. Palinski, W., Tangirala, R.K., Miller, E., Young, S.G., and Witztum, J.L. 1995. Increased autoantibody titers against epitopes of oxidized LDL in LDL receptor-deficient mice with increased atherosclerosis. Arterioscler. Thromb. Vasc. Biol. 15:1569-1576. 
19. Tangirala, R.K., Rubin, E.M., and Palinski, W. 1995. Quantitation of atherosclerosis in murine models: correlation between lesions in the aortic origin and in the entire aorta, and differences in the extent of lesions between sexes in LDL receptor-deficient and apolipoprotein E-deficient mice. J. Lipid Res. 36:2320-2328.

20. Moazed, T.C., Kuo, C., Grayston, J.T., and Campbell, L.A. 1997. Murine models of Chlamydia pneumoniae infection and atherosclerosis. J. Infect. Dis. 175:883-890.

21. Yang, Z.P., Kuo, C.C., and Grayston, J.T. 1995. Systemic dissemination of Chlamydia pneumoniae following intranasal inoculation in mice. $J$. Infect. Dis. 171:736-738.

22. Moazed, T.C., Kuo, C.C., Grayston, J.T., and Campbell, L.A. 1998. Evidence of systemic dissemination of Chlamydia pneumoniae via macrophages in the mouse. J. Infect. Dis. 177:1322-1325.

23. Nigg, C., and Eaton, M.D. 1944. Isolation from normal mice of a pneumotropic virus which forms elementary bodies. J. Exp. Med. 79:497-510.

24. Grayston, J.T., Kuo, C.C., Wang, S.P., and Altman, J. 1986. A new Chlamydia psittaci strain, TWAR, isolated in acute respiratory tract infections. $N$. Engl.J. Med. 315:161-168.
25. Zhong, G.M., Toth, I., Reid, R., and Brunham, R.C. 1993. Immunogenicity evaluation of a lipidic amino acid-based synthetic peptide vaccine for Chlamydia trachomatis. J. Immunol. 151:3728-3736.

26. Zhong, G.M., et al. 1989. Role of endogenous gamma interferon in host defense against Chlamydia trachomatis infections. Infect. Immun. 57:152-157.

27. Cotter, T.W., et al. 1995. Protective efficacy of major outer membrane protein-specific immunoglobulin A (IgA) and IgG monoclonal antibodies in a murine model of Chlamydia trachomatis genital tract infection. Infect. Immun. 63:4704-4714.

28. Su, H., and Caldwell, H.D. 1995. Kinetics of chlamydial antigen processing and presentation to $\mathrm{T}$ cells by paraformaldehyde-fixed murine bone marrow-derived macrophages. Infect. Immun. 63:946-953.

29. Zhong, G.M., and Brunham, R.C. 1991. Antigenic determinants of the chlamydial major outer membrane protein resolved at a single amino acid level. Infect. Immun. 59:1141-1147.

30. Capron, L. 1996. Chlamydia in coronary plaques-hidden culprit or harmless hobo? Nat. Med. 2:856-857.

31. Juvonen, J., et al. 1996. Immunohistochemical detection of Chlamydia pneumoniae in abdominal aortic aneurysms. Ann. NY Acad. Sci. 800:236-238. 